\title{
Novel markers for enterochromaffin cells and gastrointestinal neuroendocrine carcinomas
}

\author{
Justyna Leja ${ }^{1,6}$, Ahmed Essaghir ${ }^{2,6}$, Magnus Essand ${ }^{1,6}$, Kenneth Wester ${ }^{3}$, Kjell Öberg ${ }^{4}$, \\ Thomas H Tötterman ${ }^{1}$, Ricardo Lloyd ${ }^{5}$, George Vasmatzis ${ }^{5}$, Jean-Baptiste Demoulin ${ }^{2}$ and \\ Valeria Giandomenico ${ }^{1,4}$
}

${ }^{1}$ Division of Clinical Immunology, Rudbeck Laboratory, Uppsala University, Uppsala, Sweden; ${ }^{2}$ Université Catholique de Louvain, de Duve Institute, Brussels, Belgium; ${ }^{3}$ Department of Genetics and Pathology, The Rudbeck Laboratory, Uppsala University, Uppsala, Sweden; ${ }^{4}$ Department of Endocrine Oncology, University Hospital, Uppsala, Sweden and ${ }^{5}$ Department of Laboratory Medicine and Pathology, Mayo Clinic, Rochester, $M N, U S A$

\begin{abstract}
The gene expression profile of metastasizing serotonin-producing neuroendocrine carcinomas, which arise from enterochromaffin cells in the jejunum and ileum, is still largely unknown. The aim of this study was to identify genes and proteins, which are preferentially expressed by neuroendocrine carcinoma and enterochromaffin cells and therefore potential novel biomarkers and/or therapeutic targets. Six carcinoma specimens and six normal ileal mucosas were profiled by Affymetrix microarrays. Advanced bioinformatics identified differentially and specifically expressed genes, which were validated by quantitative real-time-PCR on tumor cells extracted by laser capture microdissection and normal enterochromaffin cells extracted by immunolaser capture microdissection. We identified six novel marker genes for neuroendocrine carcinoma cells: paraneoplastic antigen Ma2 (PNMA2), testican-1 precursor (SPOCK1), serpin A10 (SERPINA10), glutamate receptor ionotropic AMPA 2 (GRIA2), G protein-coupled receptor 112 (GPR112) and olfactory receptor family 51 subfamily E member 1 (OR51E1). GRIA2 is specifically expressed by neuroendocrine carcinoma cells whereas the others are also expressed by normal enterochromaffin cells. GPR112 and OR51E1 encode proteins associated with the plasma membrane and may therefore become targets for antibody-based diagnosis and therapy. Hierarchical clustering shows high similarity between primary lesions and liver metastases. However, chemokine C-X-C motif ligand 14 (CXCL14) and NK2 transcription factor related locus 3 Drosophila (NKX2-3) are expressed to a lower level in liver metastases than in primary tumors and normal enterochromaffin cells, which implies a role in neuroendocrine carcinoma differentiation. In conclusion, this study provides a list of genes, which possess relatively specific expression to enterochromaffin and neuroendocrine carcinoma cells and genes with differential expression between primary tumors and metastases. We verified six novel marker genes that may be developed as biomarkers and/or therapeutic targets.
\end{abstract}

Modern Pathology (2009) 22, 261-272; doi:10.1038/modpathol.2008.174; published online 24 October 2008

Keywords: gastrointestinal neuroendocrine carcinoma; bioinformatics analysis; novel markers

Gastroenteropancreatic neuroendocrine tumors have been considered as rare diseases. However, new epidemiological studies have revealed that their prevalence has increased significantly during the last 30 years. $^{1}$ They were originally named

Correspondence: Dr V Giandomenico, PhD, Division of Clinical Immunology, Rudbeck Laboratory, Uppsala University, Uppsala SE-75185, Sweden.

E-mail: valeria.giandomenico@klinimm.uu.se

${ }^{6}$ These authors contributed equally to this work.

Received 1 August 2008; revised 1 September 2008; accepted 8 September 2008; published online 24 October 2008 carcinoids and divided according to their embryological origin into foregut (lung, stomach, duodenum, upper jejunum, and pancreas), midgut (lower jejunum, ileum, appendix, and cecum) and hindgut (colon, rectum) carcinoids. In 2000, the World Health Organization published a new classification using the terms neuroendocrine tumor (benign carcinoid) and neuroendocrine carcinoma (malignant carcinoid). ${ }^{2}$ The term carcinoid has however not been abandoned. Most neuroendocrine carcinoma patients have developed metastatic disease at the time of diagnosis due to the lack of sensitive and specific methods for early detection of primary 
tumors. Available treatments prolong survival but do not cure metastatic disease. Therefore, novel diagnostic and therapeutic techniques are highly warranted.

Our study focuses on metastasizing serotoninproducing, well-differentiated neuroendocrine carcinomas, which arise from enterochromaffin cells in the distal jejunum and ileum, tumors that often metastasize to the liver. They are also known as classical midgut carcinoids. Enterochromaffin cells derive from the diffuse neuroendocrine system and secrete a number of biologically active polypeptides and amines of which serotonin is the most prominent. Excessive secretion of serotonin from large liver metastases of neuroendocrine carcinomas may give rise to the so-called carcinoid syndrome, which include diarrhea, flushing, bronchial constriction, wheezing and valvular heart disease. ${ }^{3}$ The complex nature of enterochromaffin cell function and the limited access to biopsy material from neuroendocrine carcinomas have delayed the elucidation of the molecular genetics and tumor biology. However, recent advancements in neuroendocrine carcinoma tumor biology have moved the research studies from purely academic to studies including translational and clinical applications. ${ }^{4-10}$ Furthermore, recent development in cancer gene therapy, virotherapy and immunotherapy ${ }^{11-13}$ imply that such approaches may be applied to metastatic neuroendocrine carcinoma after identification of specifically expressed genes and target antigens.

Expression profiling using microarray analysis has been used to investigate different malignancies such as colon carcinoma, pancreatic endocrine tumors, non-small-cell lung carcinoma, breast carcinoma, and melanoma. ${ }^{14-19}$ It has also been used to investigate normal and neoplastic human enterochromaffin cells ${ }^{4,20}$ and recently gastrointestinal (GI) neuroendocrine tumors. ${ }^{21}$ However, the gene profile of metastasizing serotonin-producing neuroendocrine carcinomas is still partly unknown. Therefore, we set out to investigate the gene expression profiles of neuroendocrine carcinoma on Affymetrix microarrays and applied advanced bioinformatics to identify specifically and differentially expressed genes that can become novel markers and therapeutic molecular targets.

\section{Materials and methods}

\section{Tissue Samples}

Snap-frozen tissue blocks from 11 men and 16 women, which had a histopathologically confirmed diagnosis of neuroendocrine carcinoma, were included in the study, Table 1 . We selected three primary tumors (P1, P2, P3) and three liver metastases (L1, L2, L3) with a tumor cell population higher than $80 \%$ and we used three frozen specimens from normal ileal mucosa (N1, N2, N3) and three ileal mucosal scrapings (S1, S2, S3) as normal tissue references. Permission to collect tumor specimens was approved by the regional Ethical Committee at the Uppsala University Hospital (ref. no.Ups 02-077).

\section{Total RNA Preparation and GeneChip Hybridization onto Affymetrix Arrays}

The 12 specimens were cut in $20 \mu \mathrm{m}$ sections by using a microtome cryostat (Microm, Walldorf, Germany). Total RNA (3-5 $\mu$ g for each specimen) was prepared by using the RNeasy Mini Kit (Qiagen, Hilden, Germany) and verified by using the RNA 6000 Nano kit and the Agilent 2100 Bioanalyzer (Agilent Technologies, Santa Clara, CA, USA). About $1.5 \mu \mathrm{g}$ of total RNA per specimen were sent to the Swegene DNA Microarray Resource Centre, Lund University, Lund, Sweden, where samples were hybridized onto the Affymetrix Human Genome U133 Plus 2.0 GeneChips (Affymetrix, Santa Clara, CA, USA) and processed according to Affymetrix technical protocols.

\section{Microarray Data Analysis and Data Mining}

Scanned images of microarray chips were analyzed by the GeneChip Operating Software (Affymetrix). The arrays were normalized by $\log _{2}$ transformation, using the MAS5 Statistical Algorithm Software Developers Kit (chip-to-chip) and Z-Scores normalization on the gene level (gene-to-gene). A hierarchical clustering algorithm, Unweighted Pair-Group Method with Arithmetic mean (UPGMA), was applied to group genes and samples based on similarities in expression. Significance analysis of microarrays (SAM) was performed to identify differentially expressed genes. The analysis was performed using the TM4 system, ${ }^{22}$ taking into account four classes of samples (N, S, P, L). Differentially expressed genes were clustered according to Euclidian distance (genes and samples). Gene function within each cluster was analyzed based on gene ontology (GO) using David and EASE (http://david.abcc.ncifcrf.gov).

A specialized bioinformatics method was applied to identify tissue-specific genes that encode cell membrane proteins. The method quantifies tissue specificity by accumulation of expressed sequence tags (ESTs) corresponding to distinct genes by mapping to the human genome sequence. A numeric value of specificity of a gene for a particular tissue is calculated by an algorithm, which compares the normalized number of ESTs in that tissue with the EST number in all normal and cancer tissues. ${ }^{23}$ All Affymetrix probe-set sequences were mapped on the human genome and were assigned specificity according to the cluster they belonged to. We first excluded all probes with max intensity less than 400 for all samples, which left about 8700 probes. All probes that had ESTs from more than 7 tissues were 
Table 1 Serotonin-producing neuroendocrine carcinoma specimens

\begin{tabular}{|c|c|c|c|c|c|}
\hline Patient & Sex/age ${ }^{\mathrm{a}}$ & Carc. syndrome & Treatment & Tumor type & Tissue \\
\hline 1 (P1) & $\mathrm{M} / 70$ & - & Untreated & Primary tumor & Ileum \\
\hline $1 \mathrm{a}$ & $\mathrm{M} / 70$ & - & Untreated & Primary tumor & Ileum \\
\hline $1 b$ & $\mathrm{M} / 70$ & - & Untreated & Metastasis & Mesothelium \\
\hline $2(\mathrm{P} 2)$ & $\mathrm{M} / 80$ & + & Untreated & Primary tumor & Ileocecum \\
\hline 3 & $\mathrm{~F} / 68$ & + & Untreated & Primary tumor & Ileum \\
\hline 4 & $\mathrm{M} / 50$ & + & Untreated & Primary tumor & Ileum \\
\hline 5 & $\mathrm{~F} / 64$ & - & Untreated & Primary tumor & Ileum \\
\hline $5 a$ & $\mathrm{~F} / 64$ & - & Untreated & Metastasis & Liver/omentum \\
\hline 6 & $\mathrm{M} / 71$ & - & Untreated & Primary tumor & Jejunum \\
\hline 7 & $\mathrm{~F} / 76$ & - & Untreated & Primary tumor & Ileum \\
\hline 8 & $\mathrm{~F} / 63$ & - & Untreated & Primary tumor & Ileum \\
\hline 9 & $\mathrm{~F} / 67$ & + & Untreated & Primary tumor & Ileum \\
\hline 10 & $\mathrm{~F} / 56$ & - & Untreated & Primary tumor & Ileum \\
\hline 11 (P3) & $\mathrm{F} / 68$ & + & Untreated & Primary tumor & Ileum \\
\hline $11 \mathrm{a}$ & $\mathrm{F} / 68$ & + & Untreated & Primary tumor & Ileum \\
\hline $11 \mathrm{~b}$ & $\mathrm{~F} / 68$ & + & Untreated & Metastasis & Mesothelium \\
\hline 12 & $\mathrm{M} / 77$ & + & Untreated & Primary tumor & Ileum \\
\hline 13 & $\mathrm{~F} / 43$ & + & Untreated & Primary tumor & Ileum \\
\hline 14 & $\mathrm{~F} / 77$ & - & IFN & Primary tumor & Ileum \\
\hline 15 (L1) & $\mathrm{M} / 52$ & + & SMS, IFN & Metastasis & Liver \\
\hline $15 a$ & $\mathrm{M} / 52$ & + & SMS, IFN & Metastasis & Liver \\
\hline 16 (L2) & $\mathrm{F} / 64$ & + & SMS, IFN & Metastasis & Liver \\
\hline $16 \mathrm{a}$ & $\mathrm{F} / 64$ & + & SMS, IFN & Metastasis & Liver \\
\hline 17 (L3) & $\mathrm{F} / 75$ & + & SMS, IFN & Metastasis & Liver \\
\hline 18 & $\mathrm{M} / 54$ & + & SMS, IFN & Metastasis & Mesothelium \\
\hline 19 & $\mathrm{M} / 51$ & + & SMS & Metastasis & Mesothelium \\
\hline 20 & $\mathrm{~F} / 50$ & + & Untreated & Metastasis & Mesothelium \\
\hline 21 & $\mathrm{M} / 56$ & + & Untreated & Metastasis & Liver \\
\hline 22 & $\mathrm{~F} / 52$ & + & SMS, IFN & Metastasis & Mesothelium \\
\hline 23 & $\mathrm{~F} / 44$ & - & SMS, IFN & Metastasis & Mesothelium \\
\hline 24 & $\mathrm{M} / 41$ & - & SMS, IFN & Metastasis & Mesothelium \\
\hline 25 & $\mathrm{~F} / 40$ & + & SMS, IFN & Metastasis & Liver \\
\hline 26 & $\mathrm{M} / 52$ & + & SMS, IFN & Metastasis & Liver \\
\hline 27 & $\mathrm{~F} / 70$ & + & $\mathrm{SS}, \mathrm{IFN}$ & Metastasis & Mesothelium \\
\hline
\end{tabular}

SMS, somatostatin; IFN, interferon; SS, sandostatin; M, male; F, female; Carc., carcinoid.

${ }^{\text {a }}$ Age at the time of operation.

then excluded, leaving only 161 probes. Probes that had words 'membrane' or 'receptor' in their GO terms (Affymetrix annotation file, September 2006) were selected and this reduced the list to 50 probes. product covered at least one exon-exon junction, Supplementary Table 1. The PCR reactions were analyzed on agarose gels and quantified by NIH Image 1.62 software.

\section{PCR Analysis using Commercial cDNA}

PCR analyses, on commercially available cDNA from normal tissues (Clontech BD Biosciences, Palo Alto, CA, USA), were performed to investigate 14 genes: glutamate receptor, ionotropic, AMPA 2 (GRIA2), PTPRN, APLP1, ST18, SPOCK1, SCG3, paraneoplastic antigen Ma2 (PNMA2), serpin A10 (SERPINA10), chemokine (CXC motif) ligand 14 (CXCL14), NK2 transcription factor related, locus 3, Drosophila (NKX2-3), G protein-coupled receptor 112 (GPR112), $K L B$, olfactory receptor, family 51, subfamily E, member 1 (OR51E1) and TLE2, bold in Table 2. $\beta$-Actin was used as a positive and quantitative control. All primers were designed de novo using the Primer Express Software Version 3.0 Software (Applied Biosystems, Foster City, CA, USA) to amplify target cDNA only, ie, each PCR

\section{Laser Capture Microdissection of Normal Epithelial Cells of Ileal Mucosa and Neuroendocrine Carcinoma Cells}

Laser capture microdissection can be used to obtain single cells. Snap-frozen specimens (three $\mathrm{N}$, three $\mathrm{P}$, three L) were cut in $10 \mu \mathrm{m}$ sections by using a microtome cryostat (Microm) and adhered to polyethylene-naphtalate membrane slides (Carl Zeiss, Stockholm, Sweden). Normal epithelial cells of ileal mucosa and tumor cells from primary tumors and liver metastases were isolated by using the PALM $^{\mathbb{R}}$ Robot Microbeam laser microdissection system (P.A.L.M Microlaser Technologies, Bernried, Germany) according to the procedure described by Micke et al. ${ }^{24}$ Approximately 5000 cells were collected from each section. 
Table 2 Selected genes for microarray analyses validation

Expression in tumors vs normal tissues

\begin{tabular}{|c|c|c|c|c|}
\hline Symbol & Description & Ratio & $\log _{2}$ ratio & Ensembl ID \\
\hline \multicolumn{5}{|c|}{ Genes highly expressed in tumor tissues; identified by SAM } \\
\hline GRIA2 & Glutamate receptor, ionotropic, AMPA2 & 1317 & 10.4 & ENSG00000120251 \\
\hline PTPRN & Protein tyrosine phosphatase & 206 & 7.7 & ENSG00000054356 \\
\hline APLP1 & Amyloid- $\beta$ precursor-like protein 1 & 180 & 7.5 & ENSG00000105290 \\
\hline ST18 & Suppression of tumorigenicity 18 & 143 & 7.2 & ENSG00000147488 \\
\hline SPOCK1 & Sparc, osteonectin (proteoglycan) & 141 & 7.1 & ENSG00000152377 \\
\hline C19orf30 & Chromosome 19 open-reading frame 30 & 140 & 7.1 & ENSG00000176840 \\
\hline NMNAT2 & Nicotinamide nucleotide adenylyltransferase 2 & 134 & 7.1 & ENSG00000157064 \\
\hline САМК $2 B$ & $\mathrm{Ca} / c a l m o d u l i n-d e p e n d e n t$ protein kinase II- $\beta$ & 129 & 7.0 & ENSG00000058404 \\
\hline F5 & Coagulation factor $\mathrm{V}$ & 126 & 7.0 & ENSG00000198734 \\
\hline KIF1A & Kinesin family member $1 \mathrm{~A}$ & 123 & 6.9 & ENSG00000130294 \\
\hline KIRREL2 & Kin of IRRE-like 2 & 118 & 6.9 & ENSG00000126259 \\
\hline ACTL6B & Actin-like 6B & 112 & 6.8 & ENSG00000077080 \\
\hline PRODH2 & Proline dehydrogenase 2 & 106 & 6.7 & ENSG00000161270 \\
\hline F5 & Coagulation factor $\mathrm{V}$ & 87 & 6.4 & ENSG00000198734 \\
\hline SLC8A2 & Sodium-calcium exchanger & 83 & 6.4 & ENSG00000118160 \\
\hline INA & Internexin neuronal intermediate filament protein, $\alpha$ & 79 & 6.3 & ENSG00000148798 \\
\hline SCG3 & Secretogranin III & 77 & 6.3 & ENSG00000104112 \\
\hline GABRB3 & $\gamma$-Aminobutyric acid A receptor, $\beta$-3 & 77 & 6.3 & ENSG00000166206 \\
\hline$R P R M L$ & Reprimo-like & 75 & 6.2 & ENSG00000179673 \\
\hline NRCAM & Neuronal cell adhesion molecule & 75 & 6.2 & ENSG00000091129 \\
\hline KIAA1409 & KIAA1409 & 71 & 6.1 & ENSG00000133958 \\
\hline B3GAT1 & $\beta$-1,3-Glucuronyltransferase 1 & 67 & 6.1 & ENSG00000109956 \\
\hline PNMA2 & Paraneoplastic antigen MA2 & 66 & 6.1 & ENSG00000171362 \\
\hline SERPINA10 & $\alpha-1$ Antiproteinase, antitrypsin & 66 & 6.0 & ENSG00000140093 \\
\hline \multicolumn{5}{|c|}{ Genes downregulated in carcinoid liver metastases compared to primary tumors; identified by SAM } \\
\hline CXCL14 & Chemokine (CXC motif) ligand 14 & 1.20 & 0.27 & ENSG00000145824 \\
\hline NKX2-3 & NkX-2.3 transcription factor related, locus 3 & 0.33 & -1.58 & ENSG00000119919 \\
\hline \multicolumn{5}{|c|}{ Genes identified to be differentially expressed by EST database analysis of microarray data } \\
\hline GPR112 & G protein-coupled receptor 112 & & & ENSG00000156920 \\
\hline$K L B$ & Klotho- $\beta$ & & & ENSG00000134962 \\
\hline OR51E1 & Olfactory receptor, family 51 , subfamily $E$, member 1 & & & ENSG00000180785 \\
\hline TLE2 & Transducin-like enhancer of split 2 (E(sp1) homolog, Drosophila & & & ENSG00000065717 \\
\hline
\end{tabular}

SAM, significance analysis of microarrays.

Highlighted genes are further analyzed.

\section{Immunolaser Capture Microdissection of Normal Enterochromaffin Cells}

Laser capture microdissection is even more powerful when it is combined with immunocytochemical staining using specific antibodies to recognize the singular cell of interest. This technique is called immunolaser capture microdissection. Normal ileal mucosa tissue blocks were cut in $10 \mu \mathrm{m}$ (cryostat) sections and adhered on uncharged glass slides and single cells were isolated as described previously. ${ }^{8}$ Briefly, the slides were incubated in anti-chromogranin A (CgA) antiserum, washed and incubated with a secondary HRP-conjugated antibody, washed and counterstained with hematoxylin. All steps were performed at the minimum time required and always in the presence of RNAse inhibitor. A number of proteins can be used to identify normal enterochromaffin cells, eg, CgA, serotonin and vesicular monoamine transporter 1. However, not all antibodies are appropriate for immunolaser capture microdissection. We have verified in the past that our anti-CgA antiserum can be combined with laser capture microdissection. Enterochromaffin cells locate the crypts within the ileum and we estimate that one endocrine cell and 3-4 nonendocrine cells are captured with each laser treatment, ie, approximately $25 \%$ of the captured cells are endocrine and the majority of them are enterochromaffin cells. Immunostained cells were isolated using a PixCell II laser-capture microdissection system (Arcturus Bioscience, Mountain View, CA, USA). ${ }^{8}$

\section{RNA Extraction from Specimens, Laser Capture Microdissected and Immunolaser Capture Microdissected Cells}

Total RNA was isolated from snap-frozen specimens; 13 normal ileum, 15 primary tumors, 17 metastases, including the 12 specimens used for microarray analyses. Total RNA was also prepared from laser capture microdissected normal epithelial and laser capture microdissected tumor cells by using the Pico Pure RNA isolation kit (Arcturus Bioscience). 
Furthermore, total RNA was prepared from immunolaser capture microdissected normal enterochromaffin cells by using TRIzol reagent (Invitrogen, Carlsbad, CA, USA). RNA quality and quantity was verified by using the RNA 6000 pico kit and the Agilent 2100 Bioanalyzer (Agilent Technologies). RNA samples were subjected to cDNA synthesis as previously described. ${ }^{6,25}$

\section{Quantitative Real-Time-PCR}

The cDNAs were analyzed for expressions of the selected genes, bold in Table 2, using primers described in Supplementary Table 1. Gene-specific PCR products were continuously measured by the iCycler IQ real-time detection system (Bio-Rad) during 40 cycles with iQ SYBR Green Supermix (Bio-Rad). Each PCR reaction contained $2 \mathrm{ng}$ of cDNA. Quantitative values were obtained from the threshold cycle number $\left(C_{\mathrm{t}}\right)$ and the fold change in expression was evaluated using the $\Delta \Delta C_{\mathrm{t}}$ method. ${ }^{5,26}$ Target genes were normalized against $\beta$-actin from each individual sample. Average expression with standard deviation from triplicate samples was plotted in relation to average expression of $\beta$-actin (set to 100).

\section{Immunohistochemistry}

Formalin-fixed paraffin-embedded tumor material from six patients with histopathologically diagnosis of metastasizing serotonin-producing neuroendocrine carcinoma and six samples of normal ileum were obtained as $3.5 \mu \mathrm{m}$ sections from the Pathology Biobank, Uppsala University Hospital. We selected both primary tumors and metastases from all six patients. Slides were baked for $45 \mathrm{~min}, 60^{\circ} \mathrm{C}$, deparaffinized in xylene, hydrated in graded alcohols and blocked for endogenous peroxidase in $0.3 \%$ hydrogen peroxide diluted in $80 \%$ ethanol. For antigen retrieval, slides were immersed in a Decloaking chamber ${ }^{\circledR}$ (Biocare Medical, Walnut Creek, CA, USA) and boiled in citrate buffer ${ }^{\circledR}$, pH6 (Lab Vision, Freemont, CA) for 4 min $125^{\circ} \mathrm{C}$ and then allowed to cool down to $90^{\circ} \mathrm{C}$. For each antibody we used a standardized protocol for tissue microarrays, ${ }^{27,28}$ containing positive as well as negative tissues. For SCG3, we used small intestine (ileum) as positive control and prostate as negative control; for PNMA2, we used stomach as positive control and ovary as negative control; for GRIA2, we used brain (cerebellum) as positive control and intestine (duodenum) as negative control; for OR51E1, we used prostate cancer as positive control and skeletal muscle as negative control. Primary rabbit antibodies were diluted in Antibody diluents ${ }^{\circledR}$ (Lab Vision) as follows, anti-SCG3 1:250, anti-PNMA2 1:350, anti-GRIA2 1:20 (Atlas Antibodies, Stockholm, Sweden), anti-GluR-2 (GRIA2) 1:40 (Epitomics, Burlingame, CA, USA), anti-POGR
(OR51E1) 1:150 (GenWay Biotech, San Diego, CA, USA) and anti-OR51E1 1:500 (Osenses, Flagstaff Hill, Australia). Automated immunohistochemistry was carried out using an Autostainer 480 instrument ${ }^{\circledR}$ (Lab Vision). Primary antibodies and a dextran polymer visualization system (UltraVision LP HRP polymer ${ }^{\mathbb{R}}$, Lab Vision) were incubated for 30 min each at room temperature and slides were developed for $2 \times 5 \mathrm{~min}$ using Diaminobenzidine (Lab Vision) as chromogen, each incubation was followed by rinsing in wash buffer ${ }^{\circledR}$ (Lab Vision). Slides were counterstained in Mayer's hematoxylin (Histolab, Gothenburg, Sweden) and mounted by using Pertex ${ }^{\circledR}$ (Histolab).

\section{Results}

\section{Cluster Analysis Reveals Similarity between Primary} Tumor and Liver Metastasis Transcriptomes

Three primary tumors (P1, P2, P3), three liver metastases (L1, L2, L3), three normal ileal mucosa specimens (N1, N2, N3) and three ilea mucosal scrapings (S1, S2, S3) were profiled using Affymetrix microarrays. Enterochromaffin cells do not form macroscopically discrete organs and it is difficult to identify and collect enough normal enterochromaffin cells to perform microarray analysis. The closest normal cells are the epithelial cells in the ileal mucosa, which we used as reference tissue. ANOVA analyses showed a significant similarity between normal samples (N, S) and a significant similarity between tumor samples (P, L), whereas significant differences were identified between normal and tumor samples (data not shown). UPGMA hierarchical clustering was applied to group genes and samples based on expression similarities. These analyses confirmed the similarity between primary tumor and liver metastasis transcriptomes, where only L1 showed significant differences (Supplementary Figure 1). The results from the microarray analysis have been deposited on NCBI's GEO (accession number: GSE9576) and EBI's Array-Express database (accession number: E-TABM-389).

\section{Significance Analysis of Microarrays Analysis and EST Database Analysis Identify Differentially Expressed Genes between Normal and Tumor Material}

Significance analysis of microarrays analysis revealed significant differential expression of 383 probe sets (370 genes) between normal samples (N, S) and tumor samples (P, L). Genes were clustered according to Euclidian distance and their functions were analyzed using gene ontology at EASE in six independent clusters (Figure 1). The ratio of the average signals indicates that 105 probe sets (94 genes) are expressed to a higher level and 278 probe 
sets (276 genes) are expressed to a lower level in tumor material compared to normal tissue (Supplementary Table 2). We analyzed biological
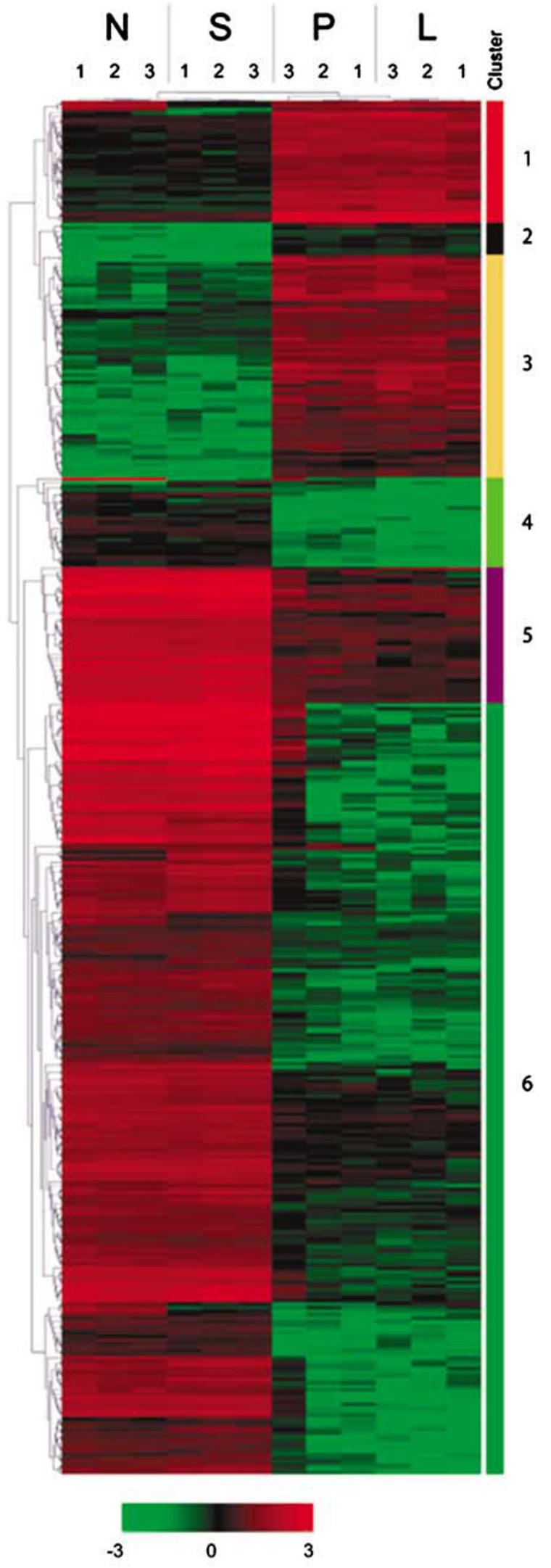

process, molecular function, and cellular component description and the GO classifiers for the 94 genes that are expressed to a higher level in tumors (Supplementary Figure 2).

A total of 30 differentially expressed genes are shown in Table 2. Twenty-four genes, upper section of Table 2, were indicated to be highly expressed in tumor specimens by SAM analysis. Although primary tumors and liver metastases transcriptomes are strikingly similar, SAM analysis identified two genes, which are potentially downregulated in metastases: CXCL14 and homeobox gene Nkx-2.3 (NKX2-3), middle section of Table 2. The specialized bioinformatics method to investigate tissue specificity of gene expression by combining the EST data base and our microarray data and identified 53 restricted probe sets (50 genes) that encode proteins associated with cell membrane (Supplementary Table 3). We selected four of those genes, lower section of Table 2. Besides in neuroendocrine carcinoma, GPR112 was expressed in normal fetal retina; $K L B$ in normal testis and mammary gland; OR51E1 in normal prostate and in colon, lung and prostate cancers; TLE2 in normal brain, testis and placenta and in breast and germ cell cancers. Fourteen genes, given in bold in Table 2, were further analyzed.

\section{PCR Analysis on cDNA from Vital Tissues Confirms Restriction in Gene Expression}

Twelve genes identified by microarray analysis to be preferentially expressed in neuroendocrine carcinoma cells: GRIA2, PTPRN, APLP1, ST18, testican-1 precursor (SPOCK1), SCG3, PNMA2, SERPINA10, GPR112, KLB, OR51E1 and TLE2 and two genes identified to be downregulated in liver metastasis: CXCL14 and NKX2-3 were first investigated for expression in normal tissues. PCR was performed on commercial cDNA from a number of vital human organs (Supplementary Table 4) and tissues of the GI tract (Supplementary Table 5). GPR112 and OR51E1 were not detected in any of the investigated organs or tissues. GRIA2, SPOCK1 and PNMA2 were only detected in brain, SERPINA10 only in liver and $K L B$ only in pancreas. SCG3 also showed restricted expression and was only detected in brain, pancreas and most GI tract tissues. APLP1, ST18 and TLE2 had a fairly ubiquitous expression pattern and were therefore excluded from further analyses. For the two genes differently expressed in primary tumors

Figure 1 Significance analysis of microarrays verified significant differential expression of 370 genes between normal and tumor samples. Theoretical number of false-positive point $=0$ and $Z$ scores were calculated based on normalized $\log _{2}$ data. Genes were clustered according to Euclidian distance in six independent clusters, as indicated in the figure. Each column represents a sample and each row represents a gene. Red, upregulation; green, downregulation. 

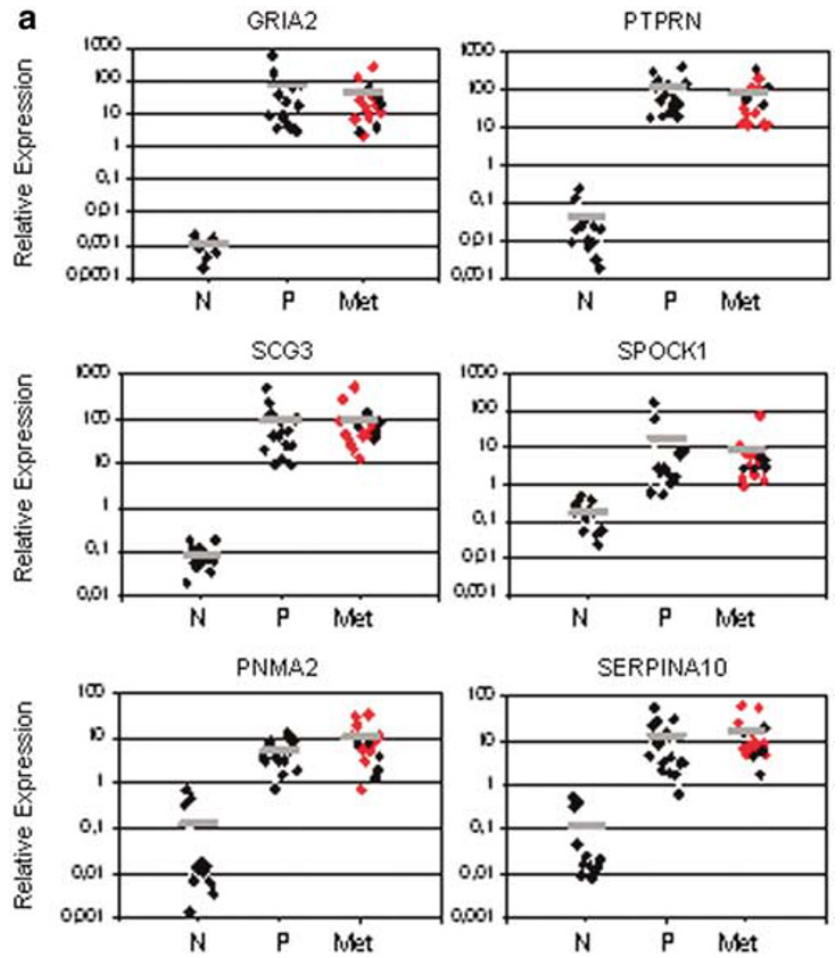

b
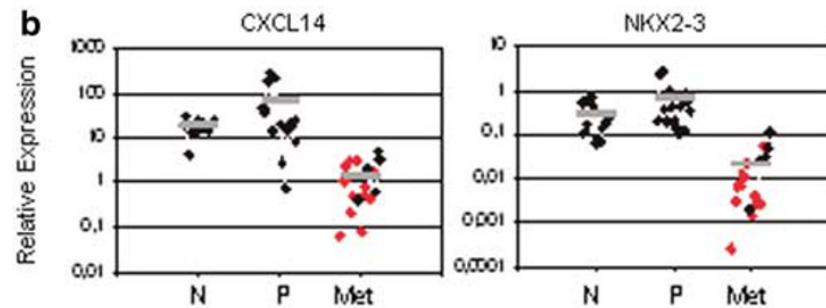

C
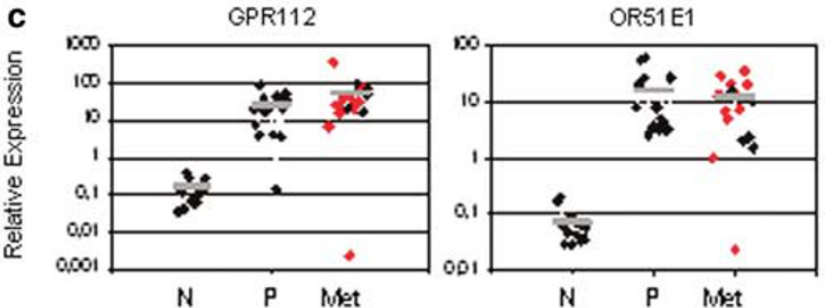

Figure 2 Quantitative real-time-PCR on normal ileal mucosa, primary tumor, liver and mesentery metastases specimens confirms microarray gene expression data. Total RNA was isolated from frozen tissue: 13 normal ileal mucosa $(\mathrm{N}), 15$ primary tumors (P) and 17 metastases (Met) (8 mesentery and 9 liver). The metastases were obtained from treated (red) and untreated (black) patients. cDNA was synthesized and Q-RT-PCR was performed for GRIA2, PTPRN, SCG3, SPOCK1, PNMA2, SERPINA10 (a); CXCL14, NKX2-3 (b); GPR112, OR51E1 (c). $C_{\mathrm{t}}$ values were calculated and the data were evaluated using the $2^{-\Delta \Delta C_{\mathrm{t}}}$ method using $\beta$-actin from each individual sample for normalization. Average values from triplicate samples with standard deviations are shown.

and liver metastases, NKX2-3 was expressed in kidney, pancreas, jejunum, cecum and transversal colon whereas CXCL14 was ubiquitously expressed.
Quantitative Real-Time-PCR Analyses on Specimens and Microdissected Cells Confirms Six Novel Marker Genes and Two Genes Downregulated in Liver Metastasis

To verify gene expression of the selected differentially expressed genes quantitative real-time (Q-RT)PCR analyses were conducted on total RNA from normal and tumor specimens (Figure 2). We found that GRIA2 is on average 10000 - to 100000 -fold more expressed in primary tumor $(\mathrm{P})$ and liver metastasis (L) than in normal ileum $(\mathrm{N})$, whereas PTPRN and SCG3 are approximately 1000-fold and SPOCK1, PNMA2 and SERPINA10 approximately 100 -fold more expressed in tumor material (Figure 2a). No differences in expression were observed between primary tumors and liver metastases. We next analyzed the expression of CXCL14 and NKX23 , which by microarray analysis were indicated to be downregulated in liver metastases. We found that $\mathrm{L}$ express both genes 10- to 100-fold less than $\mathrm{P}$ and $\mathrm{N}$ (Figure 2b). GPR112 and OR51E1 are on average approximately 100-fold more expressed in $\mathrm{P}$ and $\mathrm{L}$ than in $\mathrm{N}$ (Figure 2c), whereas $K L B$ was only slightly more expressed in tumor material than in normal tissue (data not shown). We did not detect any difference in gene expression in the metastasis specimens between treated and untreated patients.

Furthermore, Q-RT-PCR analyses were conducted on total RNA from laser capture microdissected epithelial cells of normal ileal mucosa, immunolaser capture microdissected normal enterochromaffin cells from ileal mucosa and laser capture microdissected tumor cells from primary lesions and liver metastases (Figure 3). These analyses confirmed the specific expression of the selected genes in normal enterochromaffin cells and tumor cells with the exception of GRIA2, which is not expressed in normal enterochromaffin cells. PTPRN and SCG3 were more expressed in tumor cells than in the equivalent amount of normal enterochromaffin cells (Figure 3a), indicating that they are upregulated during the malignant transformation. CXCL14 and NKX2-3 were expressed at the same level in normal enterochromaffin and primary tumor cells and at lower levels in tumor cells from liver metastases (Figure 3b). Normal enterochromaffin cells, primary and liver metastasis tumor cells specifically express GPR112 and OR51E1 (Figure 3c).

\section{Immunohistochemistry Analysis Validates Two Proteins as Molecular Targets}

We extended our analyses of the differentially expressed genes to investigate protein expression using the six commercial antibodies presently available for immunohistochemistry anti-SCG3, anti-PNMA2, anti-GRIA2, anti-GluR-2 (GRIA2), anti-POGR (OR51E1) and anti-OR51E1. Paraffinembedded tumor sections from primary tumors and metastases were used. We verified the expres- 

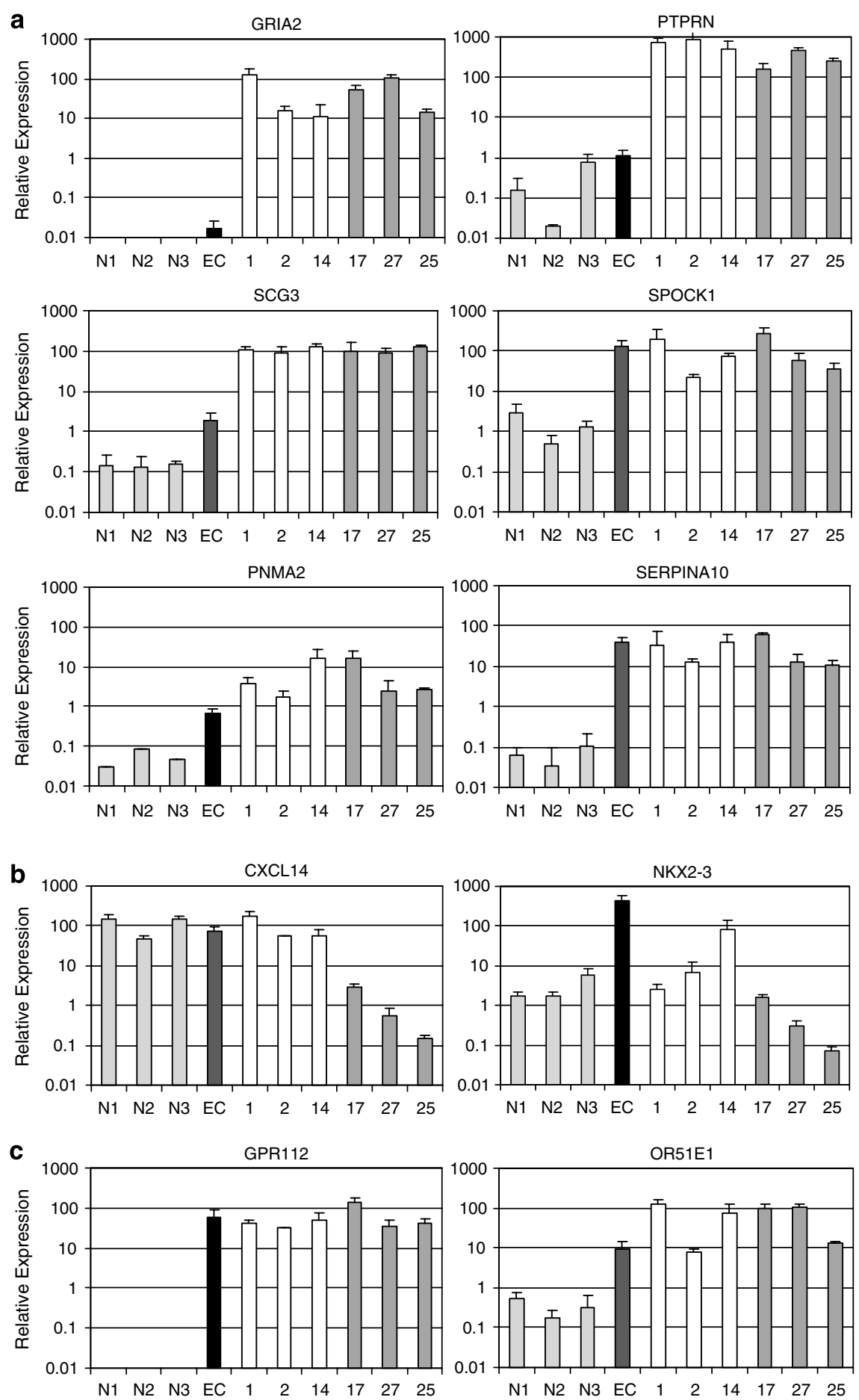

Figure 3 Quantitative real-time-PCR on microdissected normal epithelial cells from ileal mucosa, normal enterochromaffin cells, primary neuroendocrine carcinoma cells and neuroendocrine carcinoma cells from liver metastases. Total RNA was isolated from laser capture microdissected normal epithelial cells (N1, N2, N3), immunolaser capture microdissected normal enterochromaffin cells (EC), laser capture microdissected primary neuroendocrine carcinoma cells $(1,2,14)$ and laser capture microdissected neuroendocrine carcinoma cells from liver metastases $(17,27,25)$. cDNA was synthesized and quantitative real-time-PCR was performed for GRIA2, PTPRN, SCG3, SPOCK1, PNMA2 and SERPINA10 (a); CXCL14, NKX2-3 (b); GPR112, OR51E1 (c). $C_{\mathrm{t}}$ values were calculated and the data were evaluated using the $2^{-\Delta \Delta C_{\mathrm{t}}}$ method using $\beta$-actin from each individual sample for normalization. Average values from triplicate samples with standard deviations are shown. 


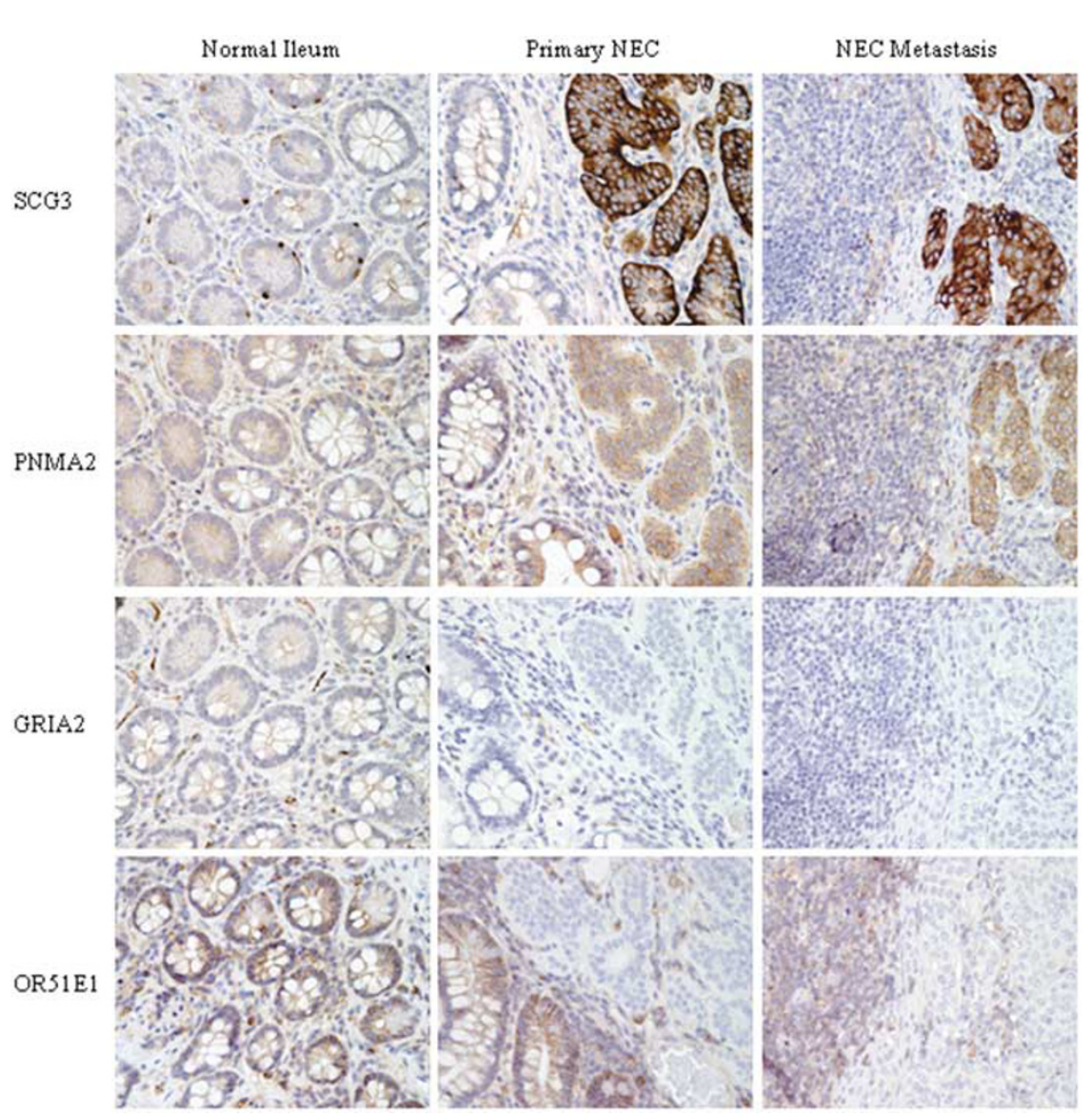

Figure 4 Immunohistochemical staining of normal ileum, primary tumor and corresponding metastasis. Secretogranin-3 (SCG3) is highly expressed exclusively in the cytoplasm of endocrine cells in normal ileum, primary tumor (six of six cases) and corresponding metastasis (six of six cases), thus verifying the carcinoid diagnosis. Paraneo-plastic antigen Ma2 (PNMA2) is weakly expressed in glandular cells but moderately expressed in primary tumor (six of six cases) and corresponding metastasis (six of six cases). Glutamate receptor 2 (GRIA2) is not expressed in neither normal nor tumor compartments. Olfactory receptor 51E1 (OR51E1) shows membranous staining in normal glandular cells and lymphoid cells but not in tumor compartments.

sion SCG3 and PNMA2 proteins both in primary tumor and liver metastases sections (Figure 4). The anti-GRIA2 antibody (Atlas Antibodies) used was not able to detect GRIA2 protein expression neither in neuroendocrine carcinoma cells of primary tumors nor in metastases (Figure 4). Furthermore, the anti-GluR2 (Epitomics) did not detect GRIA2 either (data not shown). The anti-OR51E1 (Osenses) was not able to detect OR51E1 protein expression neither in neuroendocrinoma carcinoma cells or primary tumors nor in metastases (data not shown). Anti-POGR (GenWay) showed OR51E1 expression in normal glandular cells and lymphoid cells but not in tumor compartments (Figure 4).

\section{Discussion}

This study was designed to investigate gene expression in GI neuroendocrine carcinoma cells. We analyzed primary tumors, liver and mesentery metastases using normal ileal mucosa, ileal mucosa scraping and immunolaser capture microdissected normal enterochromaffin cells as controls. The aim was at identifying genes and proteins, which possess relatively specific expression in enterochromaffin cells and neuroendocrine carcinoma cells and thereby provide a list of differentially expressed genes as a source of possible biomarkers and/or molecular targets. A further aim was at investigating differences in gene expression between primary tumors and liver metastases that might have an impact on tumor progression.

Significance analyses of microarrays analysis of our microarray data identified 94 genes with higher expression and 276 genes with lower expression in the tumor material than in the normal ileum. Gene ontology function analyses of the 94 genes with higher expression in tumor material indicates various and independent pathways, such as calcium signaling, Wnt signaling, olfactory transduction and oxidative stress that might be important for the specific neuroendocrine carcinoma cell features.

To identify potential novel biomarkers, we focused on genes previously not associated with neuroendocrine carcinoma and compared results with previously published data (Supplementary Text). The genes of interest were selected taking 
into consideration the expression levels in tumor material and the gene expression profile in normal vital organs and tissues. We propose six genes GRIA2, PNMA2, SPOCK1, SERPINA10, GPR112 and OR51E1, which may be useful diagnostic and/ or therapeutic targets. The two cell membrane proteins GPR112 and OR51E1 are truly novel markers based on tissue specificity. GRIA2, PNMA2 and SPOCK1 showed restricted expression and were only detected in normal brain whereas SERPINA10 was only detected in normal liver. The analysis on immunolaser capture microdissected normal enterochromaffin cells confirmed cell-specific gene expression of the selected genes with the exceptions of GRIA2, which was not expressed by normal enterochromaffin cells. Interestingly, we found that PTPRN and SCG3 were expressed less by normal enterochromaffin cells than by the equivalent amount of neuroendocrine carcinoma cells, indicating that the expression of these genes might be advantageous for the tumor cells.

GRIA2 encodes an AMPA glutamate receptor, which acts as an excitatory neurotransmitter at many synapses in the central nervous system. AMPA receptors are composed of four types of subunits, GluR1, GluR2, GluR3 and GluR4, which combine to form tetramers. ${ }^{29}$ The abundant GRIA2 gene expression in neuroendocrine carcinoma cells was not matched in normal enterochromaffin cells. Although the GRIA2 gene is highly expressed in tumor material, protein expression was not detected. Presumably the mRNA is not properly translated into a functional protein. GRIA2 translation can be inhibited by a polymorphic repeat sequence in the $5^{\prime}$-untranslated region. ${ }^{30}$ Additional work is needed to determine whether this is the case for neuroendocrine carcinoma cells or whether the protein in the tumor cells has been posttranslationally modified and thereby not detected by available commercial antibodies. However, the absence of GRIA2 gene expression in normal enterochromaffin cells makes GRIA2 a truly tumor-specific gene with potential as a novel diagnostic mRNA marker.

SPOCK1 encodes a modular proteoglycan, which may play a role in cell-cell and cell-matrix interactions and contribute to neuronal mechanisms in the central nervous system. It was considered to be mainly expressed in the nervous system, with a role in neural development and neuropathology. ${ }^{31} \mathrm{Re}-$ cent data have shown an involvement for SPOCK1 in glioblastoma invasion ${ }^{32}$ and prostate cancer recurrence. ${ }^{33}$ Therefore, the differential expression of this proteoglycan, which to our knowledge has never been correlated to neuroendocrine carcinoma before, is of great interest and its role in neuroendocrine carcinoma tumor invasion requires further investigation.

PNMA2 encodes the paraneoplastic antigen Ma2, which belongs to the PNMA family. Antibodies against Ma2 are often present in sera from patients suffering from paraneoplastic neurological syn- dromes. Paraneoplastic antigens, which are normally expressed only in neuronal tissues, can in the process of carcinogenesis be detected in tumors located outside the nervous system. ${ }^{34,35}$ As neuronal tissues are immunoprivileged zones, expression of these proteins in tumor cells can induce an autoimmune response with the generation of autoantibodies and/or specific cytotoxic T cells. Whether or not such immune response is a beneficial response is not fully understood. ${ }^{36}$ Our positive recognition of PNMA2 protein expression in tumor material sections both in primary tumors and metastases makes it interesting to screen whether antibodies against $\mathrm{Ma} 2$ are present in the serum of neuroendocrine carcinoma patients. This is especially so because the presence of neurological symptoms may precede the diagnosis of cancer and thereby clinicians are strongly advised to evaluate patients for this condition.

SERPINA10 encodes Serpin A10, a member of the serine protease inhibitors (serpin) superfamily that is broadly distributed. SERPINA10 expression has also been reported in endocrine pancreatic cancers. ${ }^{15}$ Considering the fact that Serpin A10 is normally produced by the liver and secreted into the plasma where it is involved in thrombosis, ${ }^{37}$ it will be of importance to evaluate the circulating levels of Serpin A10 in normal healthy individuals and neuroendocrine carcinoma patients to understand whether this protein can become a diagnostic tool for neuroendocrine carcinoma tumors. To evaluate this, we currently producing anti-serpin A10 antibodies in collaboration with the Human Protein Atlas project (http://www.proteinatlas.org).

GPR112 encodes for G protein-coupled receptor (GPCR) 112, which belongs to the adhesion family of GPCRs. GPCRs are integral membrane proteins responsible for the transduction of a remarkable diversity of endogenous signals into cellular responses. The important physiological roles of GPCR family members have led to that many of them are becoming targets for drug development. ${ }^{38,39}$ The expression of GPCRs has been correlated to various tumor types ${ }^{40}$ but, to our knowledge, this is the first study that correlates GPR112 expression to enterochromaffin cells and neuroendocrine carcinoma cells. The absence of GPR112 expression in other vital organs makes this protein a potentially novel molecular target for antibody-based therapy.

OR51E1 encodes for an odorant receptor that belongs to the olfactory receptor family, which were considered important in the olfactory epithelium. Today olfactory genes have been characterized in a number of nonolfactory tissues. ${ }^{41}$ Expression of olfactory receptors has already been correlated to normal and neoplastic enterochromaffin cells ${ }^{4,42}$ but for the first time OR51E1 is correlated to enterochromaffin and neuroendocrine carcinoma cells. Its restricted expression in normal tissue makes this gene a novel cancer biomarker. Further analyses are required to detect protein expression. 
Cluster analysis revealed that primary tumors are not clearly distinguishable from liver metastases. This finding is in line with a study on pancreatic endocrine tumors and their liver metastases. ${ }^{15}$ Although primary tumors and liver metastases transcriptomes are very similar, an important finding in our study is the deregulation of CXCL14 and NKX2-3 that are expressed to a lower level in liver metastases than in normal enterochromaffin and primary tumors, indicating involvement of these genes in metastasis formation.

CXCL14 encodes a chemokine, which belongs to the intercrine- $\alpha$ (chemokine CXC) family. Chemokines play several roles in cancer progression, including angiogenesis, inflammation, cell recruitment and migration. CXCL14 has been shown to play an inhibiting role in prostate tumor growth and it was therefore proposed that it has tumor suppressive functions. ${ }^{43}$ Furthermore, the loss of CXCL14 was correlated with low or no attraction of dendritic cells for head and neck squamous-cell carcinoma. ${ }^{44}$ More recently, CXCL14 was correlated to NK trafficking to sites of inflammation, showing that its loss might be a step in oncogenesis. ${ }^{45}$ Furthermore, CXCL14 has suppressive activity on tumor progression in oral carcinoma ${ }^{46}$ and it has with a pivotal role in the pathobiology of pancreatic cancer, probably by regulating cancer invasion. ${ }^{47}$

The NKX2-3 gene belongs to a large family of related genes that encode homeodomain-containing transcription factors. NKX2-3 encodes the transcription factor Nkx-2.3, which is involved in gut and lymphoid organs formation. ${ }^{48} \mathrm{Nkx}-2.3$ might have a role in smooth muscle carcinogenesis. ${ }^{49}$ It will therefore be of interest to evaluate whether specific changes in CXCL14 and NKX2-3 gene expression occur in patients and their possible relation with tumor stage and responsiveness to therapy.

In conclusion, we provide a comprehensive list of differentially expressed genes between normal ileal mucosa, primary neuroendocrine carcinoma tumors and neuroendocrine carcinoma liver metastases. Furthermore, we demonstrate specific expression of a small group of genes in microdissected normal enterochromaffin and neuroendocrine carcinoma cells, genes that have previously not been associated with such cells. The characterized genes may facilitate the development of molecular diagnostic screening tests and novel therapies for metastasizing serotonin-producing neuroendocrine carcinomas.

\section{Acknowledgements}

We thank Professor Emeritus Lars Grimelius, Dr Janet L Cunningham, Professor Per-Uno Malmström, Assistant Professor Manuel de la Torre and Assistant Professor Alkwin Wanders, Uppsala University Hospital for assistance with pathological examinations of samples and surgical material. We also thank Åsa Forsberg, Ulrika Larsson, Simin Tahma- sebpoor, Ulrika Johansson, Marcus Runeson, Eva Wahlund and Dr Jan Gravé for excellent technical assistance. AE and JBD were supported by grants from the Région Wallonne and from the Fonds pour la Recherche Scientifique (FNRS), Belgium. The Verto Institute, Dr Raymond and Beverly Sackler and Tore Nilsson's Foundation supported this work.

\section{Disclosure/conflict of interest}

The authors declare no conflict of interest.

\section{References}

1 Modlin IM, Oberg K, Chung DC, et al. Gastroenteropancreatic neuroendocrine tumours. Lancet Oncol 2008;9:61-72.

2 Kloppel G, Perren A, Heitz PU. The gastroenteropancreatic neuroendocrine cell system and its tumors: the WHO classification. Ann N Y Acad Sci 2004;1014:13-27.

3 Oberg K. Neuroendocrine tumors of the gastrointestinal tract: recent advances in molecular genetics, diagnosis, and treatment. Curr Opin Oncol 2005;17:386-391.

4 Modlin IM, Kidd M, Pfragner R, et al. The functional characterization of normal and neoplastic human enterochromaffin cells. J Clin Endocrinol Metab 2006;91:2340-2348.

5 Essand M, Vikman S, Grawe J, et al. Identification and characterization of a novel splicing variant of vesicular monoamine transporter 1. J Mol Endocrinol 2005;35:489-501.

6 Leja J, Dzojic H, Gustafson E, et al. A novel chromogranin-A promoter-driven oncolytic adenovirus for midgut carcinoid therapy. Clin Cancer Res 2007;13:2455-2462.

7 Vikman S, Giandomenico V, Sommaggio R, et al. CD8+ $\mathrm{T}$ cells against multiple tumor-associated antigens in peripheral blood of midgut carcinoid patients. Cancer Immunol Immunother 2008;57:399-409.

8 Nakamura N, Ruebel K, Jin L, et al. Laser capture microdissection for analysis of single cells. Methods Mol Med 2007;132:11-18.

9 Kulke MH. Gastrointestinal neuroendocrine tumors: a role for targeted therapies? Endocr Relat Cancer 2007;14:207-219.

10 Yao JC, Phan A, Hoff PM, et al. Targeting vascular endothelial growth factor in advanced carcinoid tumor: a random assignment phase II study of depot octreotide with bevacizumab and pegylated interferon alpha-2b. J Clin Oncol 2008;26:1316-1323.

11 Liu TC, Kirn D. Gene therapy progress and prospects cancer: oncolytic viruses. Gene Therapy 2008;15:877-884.

12 Douglas JT. Adenoviral vectors for gene therapy. Mol Biotechnol 2007;36:71-80.

13 Rosenberg SA, Restifo NP, Yang JC, et al. Adoptive cell transfer: a clinical path to effective cancer immunotherapy. Nat Rev Cancer 2008;8:299-308.

14 Barrier A, Lemoine A, Boelle PY, et al. Colon cancer prognosis prediction by gene expression profiling. Oncogene 2005;24:6155-6164.

15 Capurso G, Lattimore S, Crnogorac-Jurcevic T, et al. Gene expression profiles of progressive pancreatic 
endocrine tumours and their liver metastases reveal potential novel markers and therapeutic targets. Endocr Relat Cancer 2006;13:541-558.

16 Woenckhaus M, Klein-Hitpass L, Grepmeier U, et al. Smoking and cancer-related gene expression in bronchial epithelium and non-small-cell lung cancers. J Pathol 2006;210:192-204.

17 Schuetz CS, Bonin M, Clare SE, et al. Progressionspecific genes identified by expression profiling of matched ductal carcinomas in situ and invasive breast tumors, combining laser capture microdissection and oligonucleotide microarray analysis. Cancer Res 2006;66:5278-5286.

18 Talantov D, Mazumder A, Yu JX, et al. Novel genes associated with malignant melanoma but not benign melanocytic lesions. Clin Cancer Res 2005;11:7234-7242.

19 Couvelard $\mathrm{A}$, Hu J, Steers $\mathrm{G}$, et al. Identification of potential therapeutic targets by gene-expression profiling in pancreatic endocrine tumors. Gastroenterology 2006;131:1597-1610.

20 Kidd M, Modlin IM, Mane SM, et al. The role of genetic markers-NAP1L1, MAGE-D2, and MTA1-in defining small-intestinal carcinoid neoplasia. Ann Surg Oncol 2006;13:253-262.

21 Arvidsson Y, Andersson E, Bergstrom A, et al. Amyloid precursor-like protein 1 is differentially upregulated in neuroendocrine tumours of the gastrointestinal tract. Endocr Relat Cancer 2008;15:569-581.

22 Saeed AI, Sharov V, White J, et al. TM4: a free, opensource system for microarray data management and analysis. Biotechniques 2003;34:374-378.

23 Vasmatzis G, Klee EW, Kube DM, et al. Quantitating tissue specificity of human genes to facilitate biomarker discovery. Bioinformatics 2007;23:1348-1355.

24 Micke P, Ostman A, Lundeberg J, et al. Laser-assisted cell microdissection using the PALM system. Methods Mol Biol 2005;293:151-166.

25 Vikman S, Essand M, Cunningham JL, et al. Gene expression in midgut carcinoid tumors: potential targets for immunotherapy. Acta Oncol 2005;44:32-40.

26 Livak KJ, Schmittgen TD. Analysis of relative gene expression data using real-time quantitative PCR and the 2(-Delta Delta C(T)) method. Methods 2001;25:402-408.

27 Uhlen M, Ponten F. Antibody-based proteomics for human tissue profiling. Mol Cell Proteomics 2005;4:384-393.

28 Uhlen M, Bjorling E, Agaton C, et al. A human protein atlas for normal and cancer tissues based on antibody proteomics. Mol Cell Proteomics 2005;4:1920-1932.

29 Hansen KB, Yuan H, Traynelis SF. Structural aspects of AMPA receptor activation, desensitization and deactivation. Curr Opin Neurobiol 2007;17:281-288.

30 Myers SJ, Huang Y, Genetta T, et al. Inhibition of glutamate receptor 2 translation by a polymorphic repeat sequence in the $5^{\prime}$-untranslated leaders. J Neurosci 2004;24:3489-3499.

31 Charbonnier F, Perin JP, Roussel G, et al. [Cloning of testican/SPOCK in man and mouse. Neuromuscular expression perspectives in pathology]. C R Seances Soc Biol Fil 1997;191:127-133.

32 Colin C, Baeza N, Bartoli C, et al. Identification of genes differentially expressed in glioblastoma versus pilocytic astrocytoma using suppression subtractive hybridization. Oncogene 2006;25:2818-2826.
33 Wlazlinski A, Engers R, Hoffmann MJ, et al. Downregulation of several fibulin genes in prostate cancer. Prostate 2007;67:1770-1780.

34 Leyhe T, Schule R, Schwarzler F, et al. Second primary tumor in anti-Ma1/2-positive paraneoplastic limbic encephalitis. J Neurooncol 2006;78:49-51.

35 Hoffmann LA, Jarius S, Pellkofer HL, et al. Anti-Ma and anti-Ta associated paraneoplastic neurological syndromes: twenty-two newly diagnosed patients and review of previous cases. J Neurol Neurosurg Psychiatry 2008;79:767-773.

36 Eichmuller SB, Bazhin AV. Onconeural versus paraneoplastic antigens? Curr Med Chem 2007;14: 2489-2494.

37 Han X, Fiehler R, Broze Jr GJ. Isolation of a protein Z-dependent plasma protease inhibitor. Proc Natl Acad Sci USA 1998;95:9250-9255.

38 Fredriksson R, Lagerstrom MC, Hoglund PJ, et al. Novel human G protein-coupled receptors with long N-terminals containing GPS domains and Ser/Thr-rich regions. FEBS Lett 2002;531:407-414.

39 Lundstrom K. Latest development in drug discovery on G protein-coupled receptors. Curr Protein Pept Sci 2006;7:465-470.

40 Bhola NE, Grandis JR. Crosstalk between G-proteincoupled receptors and epidermal growth factor receptor in cancer. Front Biosci 2008;13:1857-1865.

41 Zhang X, De la Cruz O, Pinto JM, et al. Characterizing the expression of the human olfactory receptor gene family using a novel DNA microarray. Genome Biol 2007;8:R86.

42 Braun T, Voland P, Kunz L, et al. Enterochromaffin cells of the human gut: sensors for spices and odorants. Gastroenterology 2007;132:1890-1901.

43 Schwarze SR, Luo J, Isaacs WB, et al. Modulation of CXCL14 (BRAK) expression in prostate cancer. Prostate 2005;64:67-74

44 Shurin GV, Ferris RL, Tourkova IL, et al. Loss of new chemokine CXCL14 in tumor tissue is associated with low infiltration by dendritic cells (DC), while restoration of human CXCL14 expression in tumor cells causes attraction of DC both in vitro and in vivo. J Immunol 2005;174:5490-5498.

45 Starnes T, Rasila KK, Robertson MJ, et al. The chemokine CXCL14 (BRAK) stimulates activated NK cell migration: implications for the downregulation of CXCL14 in malignancy. Exp Hematol 2006;34: 1101-1105.

46 Ozawa S, Kato Y, Komori R, et al. BRAK/CXCL14 expression suppresses tumor growth in vivo in human oral carcinoma cells. Biochem Biophys Res Commun 2006;348:406-412.

47 Wente MN, Mayer C, Gaida MM, et al. CXCL14 expression and potential function in pancreatic cancer. Cancer Lett 2008;259:209-217.

48 Pabst O, Forster R, Lipp M, et al. NKX2.3 is required for MAdCAM-1 expression and homing of lymphocytes in spleen and mucosa-associated lymphoid tissue. EMBO J 2000;19:2015-2023.

49 Phiel CJ, Gabbeta V, Parsons LM, et al. Differential binding of an SRF/NK-2/MEF2 transcription factor complex in normal versus neoplastic smooth muscle tissues. J Biol Chem 2001;276:34637-34650.

Supplementary Information accompanies the paper on Modern Pathology website (http://www.nature.com/ modpathol) 